\title{
BYPASS GÁSTRICO LAPAROSCÓPICO COM USO REDUZIDO DE SUTURAS MECÂNICAS
}

\author{
Laparoscopic gastric bypass with low use of stapler sutures \\ Josemberg Marins CAMPOS, Eudes Paiva GODOY, Luciana Teixeira de SIQUEIRA, \\ Luis Fernando Lobato EVANGELISTA, Carolina de Souza VASCONCELOS, \\ Álvaro Antonio Bandeira FERRAZ, Edmundo Machado FERRAZ
}

ABCDDV/596

Campos JM, Godoy EP, Siqueira LT, Evangelista LFL, Vasconcelos CS, Ferraz AAB, Ferraz EM. Bypass gástrico laparoscópico com uso reduzido de suturas mecânicas. ABCD Arq Bras Cir Dig. 2008;21(2):73-6

RESUMO - Racional - A execução de bypass gástrico laparoscópico em hospital universitário público tem sido difícil devido ao elevado custo dos grampeadores cirúrgicos que prejudica o treinamento de médicos residentes e tem motivado a busca por técnicas alternativas, de baixo custo, mantendo a eficácia. Objetivo - Apresentar a viabilidade de um método com menor uso de suturas mecânicas. Métodos - Foram operados 63 pacientes em 2 hospitais universitários, sendo 12 homens e 51 mulheres (81\%), com média de 33,5 anos de idade e IMC médio de 43 . Aplicou-se a seguinte padronização técnica: Secção da alça com bisturi elétrico a $50 \mathrm{~cm}$ do ângulo duodeno-jejunal, anastomose término-lateral, passagem da alça retrocólica e retrogástrica, confecção da parede lateral da bolsa gástrica com 1 carga azul de 45 e outra de 60 mm após a secção horizontal com bisturi elétrico, sutura do estômago excluso e anastomose gastrojejunal. As anastomoses foram manuais e contínuas com fio absorvível. $\boldsymbol{R} \boldsymbol{e}$ sultados - O tempo operatório médio foi de 5,5 horas. As complicações precoces foram: fístula no ângulo de esôfago-gástrico (1,6\%), estenose $(1,6 \%)$ e fístula na anastomose gastrojejunal $(1,6 \%)$ e torção da anastomose intestinal $(1,6 \%)$. A estenose foi tratada por dilatação endoscópica e as outras complicações através de 3 re-operações (2 laparoscópicas e 1 laparotômica). O tempo de internação variou de 2 a 20 dias, com média de 4 dias, não havendo óbito. Conclusãa - Este método é viável e com baixo custo operacional; todavia, é complexo e requer habilidade principalmente em suturas laparoscópicas.

DESCRITORES - Cirurgia bariátrica. Bypass gástrico laparoscópico. Suturas mecânicas. Custo.

\section{INTRODUÇÃO}

O bypass gástrico sem grampeador ou usando menor número de cargas é procedimento que já foi descrito em poucas séries na literatura, e apresenta a vantagem de diminuir custos e complicações associadas ao uso de grampeador, tal como sangramento e fístula. Por outro lado, o tempo cirúrgico é maior, há risco de lesão térmica e a curva de aprendizado é mais longa ${ }^{1}$.

Assim, objetiva-se com a proposta deste trabalho apresentar alternativa de método com baixo custo operacional.

\section{MÉTODOS}

De agosto de 2005 a outubro de 2006, foram operados 63 pacientes no Hospital Universitário Onofre Lopes da Universidade Federal do Rio Grande do Norte - UFRN - e Hospital das Clínicas da Universidade Federal do Pernambuco - UFPE -, Brasil, sendo 12 homens e 51 mulheres $(81,0 \%)$, com média de 33,5 anos de idade e IMC médio de $43 \mathrm{~kg} / \mathrm{m}^{2}$, com as seguintes co-morbidades: hipertensão arterial sistêmica $(9,5 \%)$, diabetes mellitus (5,7\%), hipotir-

Trabalho realizado no Hospital de Clínicas da Universidade Federal de Pernambuco, Recife-PE e no Hospital Universitário Onofre Lopes da Universidade Federal do Rio Grande do Norte, Natal-RN

Endereço para correspondência: Josemberg Marins Campos - e-mail: berg@elogica.com.br eoidismo $(2,5 \%)$ e colecistolitíase $(1,8 \%)$. Os pacientes apresentavam as seguintes operações prévias: colecistectomia $(3,8 \%)$, cesárea e laqueadura tubária $(3,8 \%)$, e histerectomia $(1,6 \%)$. Foram executadas os seguintes procedimentos associados: colecistectomia $(4,7 \%)$ e gastrostomia $(3,2 \%)$.

Utilizou-se número reduzido de cargas de grampeador na confecção da bolsa gástrica, que variou de 2 a 4 cargas, enquanto que as anastomoses sempre foram manuais, apesar de algumas terem sido inicialmente confeccionadas látero-lateralmente. Paulatinamente, a experiência propiciou a redução do número de cargas e o aperfeiçoamento da tática cirúrgica, que resultou em padronização padronização técnica.

\section{Técnica operatória}

Foram colocados 6 trocartes na parede abdominal superior, sendo quatro de $5 \mathrm{~mm}, 1$ de $12 \mathrm{~mm}$ e outro de $10 \mathrm{~mm}$ (Figura 1). Os dispositivos de $5 \mathrm{~mm}$ foram distribuídos da seguinte maneira: 2 no flanco esquerdo para o cirurgiãoauxiliar, 1 abaixo do apêndice xifóide para afastar o fígado e outro subcostal direito para a mão esquerda do cirurgião. $\mathrm{O}$ trocarte de 5 a $12 \mathrm{~mm}$ foi colocado na linha mediana acima da cicatriz umbilical e o de $10 \mathrm{~mm}$ posicionado no flanco direito para passar a ótica. $\mathrm{O}$ paciente posicionava-se em decúbito dorsal sem nenhuma angulação da mesa e o monitor situado entre a cabeça e o membro superior esquerdo do paciente. 


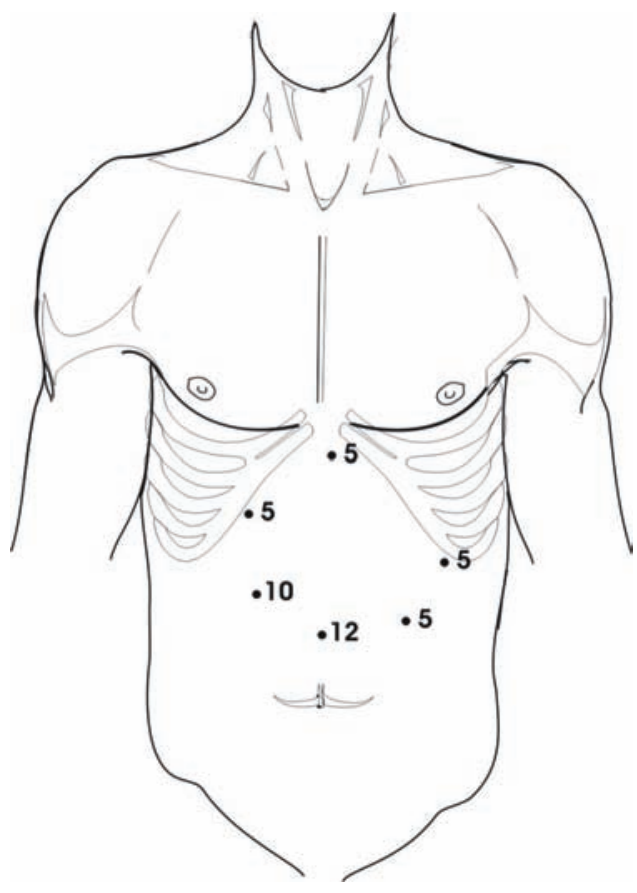

FIGURA 1 - Imagem esquemática da posição abdominal dos trocartes em bypass gástrico laparoscópico

A operação era iniciada pela confecção da anastomose intestinal, seguindo-se da bolsa gástrica. $\mathrm{O}$ auxiliar elevava o mesocólon transverso e possibilitava assim que o cirurgião realizasse as seguintes etapas: secção e fechamento da alça com bisturi elétrico a $50 \mathrm{~cm}$ do ângulo duodeno-jejunal; medição em $150 \mathrm{~cm}$, anastomose término-lateral em plano único e passagem da alça retrocólica e retrogástrica. Após o mesocólon ser colocado em posição habitual, iniciavase os passos seguintes para a confecção da bolsa gástrica: secção horizontal do estômago com bisturi elétrico, secção da parede lateral com grampeador, usando 1 carga azul de 45 e outra de 60 mm (Tyco, Boulder, CO, USA); sutura extramucosa do estômago excluso, fixação da alça na bolsa gástrica e anastomose gastro-jejunal término-lateral. O mesocólon transverso era novamente elevado para o fechamento dos espaços peritoneais com fio inabsorvível $\left(\right.$ Ethibond $^{\circledR}$ ). Todas as suturas gástricas e intestinais eram manuais e contínuas, usando fio absorvível (Caprofil ${ }^{\circledR}$ ).

A anastomose intestinal término-lateral era confeccionada a partir da inserção da extremidade biliopancreática na face lateral da alça (Figura 2). A sutura era iniciada entre o ângulo da ferida lateral e o meio da face posterior da alça biliopancreática, de maneira que a borda mesentérica dela se unisse ao meio da parede posterior. Assim, os ângulos das feridas não ficavam justapostos, o que mantinha a anastomose sempre aberta, evitando o colabamento e a obstrução por torção.

A bolsa gástrica sem anel media em torno de $6 \mathrm{~cm}$, tubular, não contendo tecido excedente ao nível do fundo gástrico. A secção lateral apresentava simetria entre a parede anterior e posterior do estômago. Para se obter este formato, após a secção horizontal passava-se fio inabsorvível com agulha $n^{\circ} 4$ (Prolene $0^{\circledR}$ ) a partir da parede posterior para uni-la com a anterior através da colocação de

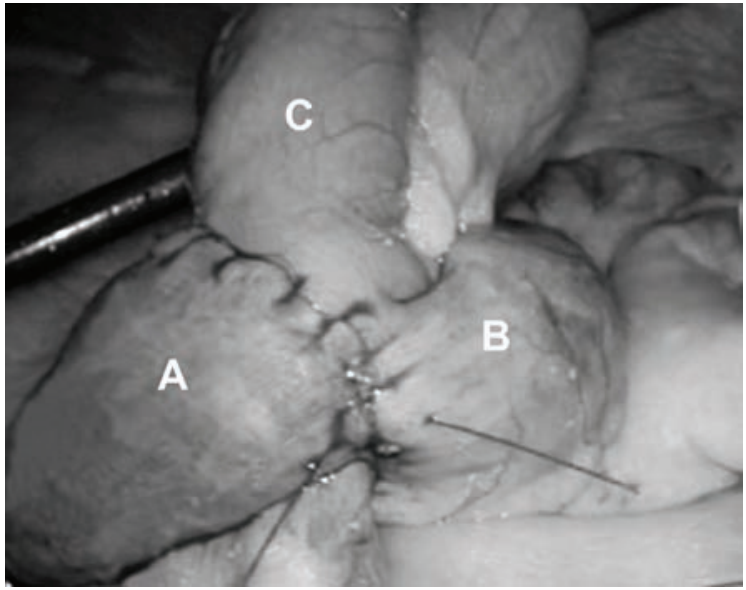

FIGURA 2 - Imagem laparoscópica da anastomose intestinal de bypass gástrico: alça biliopancreática (A), alça alimentar (B) e alça comum (C)

anteparo de borracha na extremidade do fio (Figura 3). Este fio era tracionado anteriormente para evitar a confecção de reservatório gástrico assimétrico com excesso de parede posterior. A seguir, disparava-se o grampeador com carga azul $45 \mathrm{~mm}$ entre a sonda de Fouchet previamente colocada e o referido fio de tração.

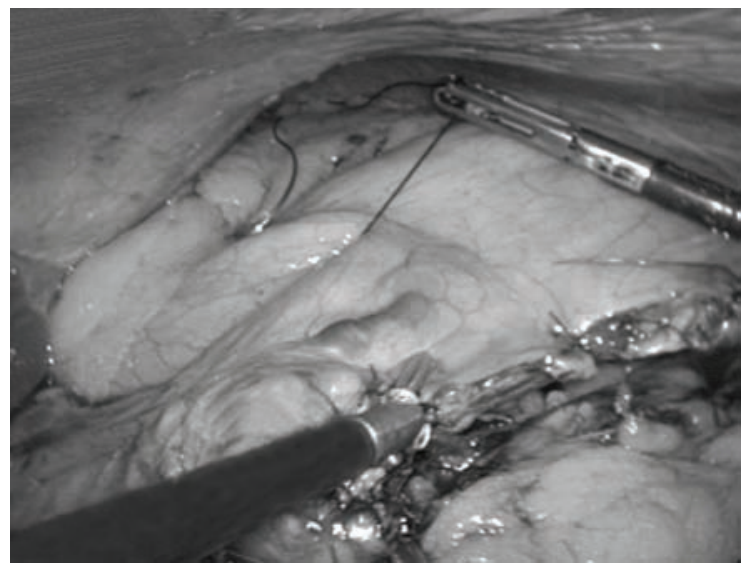

FIGURA 3 - Imagem laparoscópica da confecção do pouch mostrando tração de fio com anteparo na parede posterior do estômago após a secção horizontal do estômago

Seguindo o princípio descrito acima, passava-se o mesmo fio no fundo gástrico para também ser tracionado e facilitar a dissecção anterior e posterior do ângulo esôfagogástrico, usando pinça Maryland de ponta longa ou gold finger, que era utilizado para posicionar fita cardíaca na área a ser seccionada com o grampeador, usando carga azul de $60 \mathrm{~mm}$ (Figura 4). A tração das duas extremidades da fita facilitava o posicionamento do grampeador e evitava a inclusão de fundo gástrico em excesso, diminuindo assim o número de cargas utilizadas.

Após a fixação da alça na bolsa gástrica, a anastomose gastro-jejunal término-lateral era confeccionada com sutura extra-mucosa e plano único (Figura 5). 


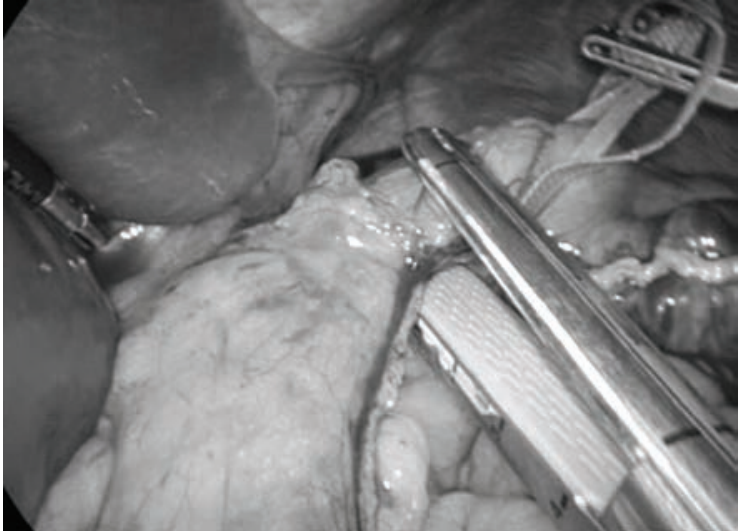

FIGURA 4 - Imagem laparoscópica mostrando fita cardíaca tracionando o fundo gástrico para facilitar a colocação da sutura mecânica

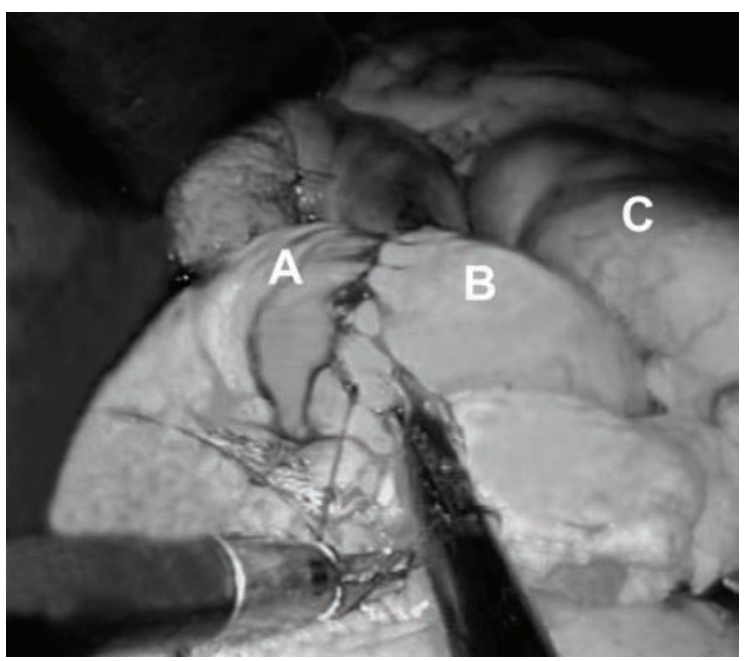

FIGURA 5 - Imagem laparoscópica da anastomose gastro-jejunal: bolsa gástrica (A), alça jejunal (B) e estômago excluso $(\mathrm{C})$

\section{RESULTADOS}

O tempo operatório médio foi de 5,5 horas e as complicações precoces foram: 1 fístula no ângulo esôfagogástrico (1,6\%), 1 estenose na anastomose gastro-jejunal $(1,6 \%), 1$ fístula na anastomose gastro-jejunal $(1,6 \%)$ e 1 torção da anastomose intestinal $(1,6 \%)$. A incidência total de fístula foi de $3,2 \%$. A estenose foi tratada por dilatação endoscópica e as outras complicações através de 3 re-operações $(4,7 \%)$, sendo 2 por via laparoscópica e 1 laparotômica (Quadro 1).

Estas re-intervenções foram realizadas precocemente apenas diante da suspeita clínica de infecção ou obstrução intracavitária, não sendo executado nenhum exame de imagem. A re-abordagem por videolaparoscopia nos 2 casos permitiu o diagnóstico precoce e facilitou o tratamento da complicação encontrada. O tempo de internação variou de 2 a 20 dias, com média de 4 dias, não havendo óbito.
QUADRO 1 - Características epidemiológicas e resultados das operações bariátricas por videolaparoscopia

\begin{tabular}{|l|l|}
\hline Sexo & $\begin{array}{l}\text { Masculino 12 }(29 \%) \\
\text { Feminino 51 (81\%) }\end{array}$ \\
\hline Operações associadas (\%) & $\begin{array}{l}\text { Colecistectomia: } 4,7 \\
\text { Gastrostomia: } 3,2\end{array}$ \\
\hline Idade (anos) & 33,5 \\
\hline IMC (kg/m $\mathbf{2})$ & 43 \\
\hline Tempo cirúrgico (horas) & 5,5 \\
\hline \multirow{3}{*}{ Complicações (\%) } & $\begin{array}{l}\text { Fístula ângulo His: } 1,6 \\
\text { Estenose anastomose gastrojejunal: } 1,6 \\
\text { Fístula anastomose gastrojejunal: } 1,6 \\
\text { Torção da anastomose intestinal: } 1,6\end{array}$ \\
\hline Reoperações & $\begin{array}{l}3 \text { (4,7\%) } \\
\text { Laparoscópicas: } 2 \\
\text { Laparotômica: } 1\end{array}$ \\
\hline Tempo de internação (dias) & $\begin{array}{l}2 \text { a 20 } \\
\text { Média }\end{array}$ \\
\hline
\end{tabular}

\section{DISCUSSÃO}

Até o momento, há poucas séries de casos na literatura usando a técnica de staplerless; o Ligasure ${ }^{\circledR}$ tem sido aplicado na confecção da bolsa gástrica e do Y de Roux, como foi apresentado pela primeira vez por Dr. Jacques Himpens no Congresso da IFSO - International Federation for the Surgery of Obesity, em 2004, no Japão ${ }^{1,3}$.

A casuística aqui apresentada tem peculiaridade do uso do bisturi elétrico monopolar durante a secção da alça jejunal, abertura da alça para realização da anastomose intestinal e a secção horizontal do estômago na confecção da bolsa gástrica. Este detalhe permite a perfeita distinção da mucosa e da seromuscular na parede intestinal, o que facilita a técnica de sutura envolvendo apenas a seromuscular, além de reduzir o custo com o menor uso de sutura mecânica.

Durante a realização de anastomose intestinal, o uso do Ligasure $^{\circledR}$ sela as camadas da parede e dificulta a execução de sutura seromuscular. Todavia, na confecção da bolsa gástrica, isto não é uma desvantagem considerando que estas áreas serão revestidas por sutura invaginante ${ }^{4,8}$. A sutura deve manter distância acima de $2 \mathrm{~mm}$ de cada lado da ferida, pois a empresa fabricante refere ocorrência de lesão térmica em torno de $2 \mathrm{~mm}$ quando usado para selar vasos 6 . Apesar das referidas vantagens, o completo equipamento de selamento Ligasure Atlas ${ }^{\mathrm{TM}}$ (Valleylab, Tyco, Boulder, CO, USA) é de custo elevado.

$\mathrm{O}$ uso de bisturi elétrico tem desvantagem relacionada à possibilidade de lesão acidental de estruturas adjacentes, além da potencial ocorrência de contaminação da cavidade com secreção entérica devido a abertura do trato gastrintestinal. A fim de evitar acidentes no momento do uso do cautério, devese observar cuidadosamente se o segmento da pinça ligada ao eletrocautério não está em contato com outras estruturas. Esses cuidados evitaram acidentes na casuística atual.

Ao longo do estudo, não se observou vazamento de secreção gastrintestinal na cavidade peritoneal a partir das alças e do estômago aberto, provavelmente em decorrência da pressão abdominal positiva do pneumoperitônio e também devido ao curto período de exposição da mucosa, que foi 
restrito ao momento da sutura de cada segmento envolvido. Isto resultou na não ocorrência de abscesso intracavitário pós-operatório.

A literatura registra controvérsias a respeito da melhor forma de realização da sutura laparoscópica, mas até mesmo o uso de sutura mecânica pode cursar com o aparecimento de isquemia e posterior ocorrência de fístula ${ }^{7}$. A comparação entre cirurgia laparotômica e laparoscópica mostrou significativa diferença a favor da técnica aberta: em mais de 25.000 pacientes submetidos a procedimento em Y de Roux, 2\% do grupo laparoscópico desenvolveram fístula, em oposição ao grupo aberto $(0.4 \%)^{5}$. No entanto, outras séries têm mostrado que esta diferença não é significativa, desde que seja superada a curva de aprendizado na cirurgia laparoscópica, cujo período é mais longo e necessita de maior número de operações².

A verdadeira incidência de fístula é difícil de ser avaliada já que existem diferentes técnicas operatórias e diversas formas de identificação deste tipo de complicação. Em estudo com mais de 6.000 pacientes submetidos a bypass gástrico laparoscópico, a taxa de fístula variou entre 0 e $4.3 \%$, não diferindo da laparotomia, que tem taxa em torno de $5.6 \%{ }^{2}$.

Nesta série, a taxa de fístula foi de $3,2 \%$, sem mortalidade, com período de internação máximo de 20 dias e havendo necessidade de internação em Unidade de Terapia Intensiva por 48 horas em apenas 1 paciente após re-operação por fístula. Isto é bom resultado considerando-se que ainda não foi ultrapassada a curva de aprendizado com este método.

A torção da anastomose intestinal ocorreu no início desta série após a realização de anastomose látero-lateral, que foi tratada através de re-operação laparoscópica, sendo realizada nova sutura anterior em sentido transverso para aumentar o diâmetro da anastomose, além de fixação da alça para evitar recidiva da obstrução. Após este episódio, as anastomoses intestinais passaram a ter a conformação término-lateral de acordo com a descrição metodológica do estudo, o que evitou a ocorrência de outros casos de torção.

A mais clara vantagem desta abordagem é a diminuição do custo do procedimento laparoscópico principalmente em nosso país, devido ao elevado valor dos grampeadores importados ${ }^{1}$. Somente assim, tem sido possível o desenvolvimento desta técnica em hospital público e principalmente em ambiente universitário, onde naturalmente deve surgir o treinamento de novas técnicas, a partir da realização de procedimentos em meio de ensino e pesquisa. Isto possibilita que cirurgiõespreceptores e residentes adquiram habilidade nestes procedimentos cirúrgicos, além de propiciar acesso da população de baixa renda à cirurgia minimamente invasiva.

O elevado tempo operatório e a longa curva de aprendizado tem sido desvantagem inicial do método, que deve contar com a presença de um tutor experiente nas primeiras operações, até que a equipe passe paulatinamente a realizar todas as etapas operatórias, associada a baixo risco de complicações inerentes ao ato. Neste estudo, estes aspectos têm sido seguidos, o que certamente permitiu a baixa morbidade e mortalidade ausente.

\section{CONCLUSÃO}

Este método é viável e com baixo custo operacional; todavia, é complexo e requer habilidade principalmente em suturas laparoscópicas.

Campos JM, Godoy EP, Siqueira LT, Evangelista LFL, Vasconcelos CS, Ferraz AAB, Ferraz EM. Laparoscopic gastric bypass with low use of stapler sutures. ABCD Arq Bras Cir Dig. 2008;21(2):73-6

ABSTRACT - Background - To perform laparoscopic gastric bypass in public university hospital has been difficult due to the high cost of the surgical staplers. This fact induced to look for different technical options, with low cost, maintaining the efficacy. Aim - To present the viability of a new method with the use of a low number of stapler devices. Methods - Sixty three patients were operated in two university hospitals, 12 men and 51 women $(81 \%)$, with mean age of $33.5 \mathrm{y}$ and average BMI of 43 . The surgical technique used followed this sequence: loop section with electrical scalpel $50 \mathrm{~cm}$ of the duodenojejunal angle; termino-lateral anastomosis; retrogastric-retrocolic passage of the Roux limb; construction of the lateral wall of the pouch using 1 blue load of 45 and other of $60 \mathrm{~mm}$ after horizontal section with electrical scalpel; suture of the excluded stomach and gastrojejunal anastomosis. The anastomoses were hand-sewn made and a single-layer continuous absorble suture was performed. Results - The average surgical time was 5.5 hours. The early complications were: fistula in the esophago-gastric angle (1.6\%), stenosis (1.6\%); fistula in the gastro-jejunal anastomosis (1.6\%); obstruction of the intestinal anastomosis (1.6\%). The stenosis was treated by endoscopic dilation. The remaining complications, with 3 re-operations ( 2 with laparoscopic and 1 with laparotomic approaches). The length of hospital stay was in average 4 days. Conclusion - This method is viable with low cost; however, it is complex and requires ability mainly in laparoscopic handsewn sutures.

HEADINGS - Bariatric surgery. Laparoscopic gastric bypass. Stapler. Cost saving.

\section{REFERÊNCIAS}

1. Ettinger JE, Ramos AC, Azaro E et al. Staplerless laparoscopic gastric bypass: a new option in bariatric surgery. Obes Surg. 2006; 16: 638-45.

2. Gonzalez R, Nelson LG, Gallhagher SF, Murr MM, Anastomotic leaks after laparoscopic gastric bypass. Obes Surg. 2004; 14: 1299-1307.

3. Himpens J. Laparoscopic Roux-en-Y gastric bypass performed without staples. Obes Surg 2004; 14: 897-7.

4. Himpens J, Leman G, Sonneville T. Laparoscopic Roux-en-Y gastric bypass performed without staples. Surg Endosc. 2005; 19: 1003.

5. Jones KBJ, Afram JD, Benotti PN, Capella RF, Cooper CG, Flanagan L, Hendrick S, Howell M, Jaroch MT, Kole K, Lirio OC, Sapala JA, Schuhknecht MP, Shapiro RP, Sweet WA, Wood MH. Open versus laparoscopic Roux-en-Y gastric bypass: a comparative study of over 25,000 open cases and the major laparoscopic bariatric reported series. Obes Surg. 2006; 16: 721-27.
6. LigaSure Vessel Sealing System ${ }^{\mathrm{TM}}$ (www.ligasure.com)

7. Lujan JA, Frutos MD, Quitéria H, Cuenca JR, Valero G. Experience with the circular stapler for the gastrojejunostomy in laparoscopic gastric bypass (350 cases). Obes Surg. 2005; 15:1096-102.

8. Ramos AC, Galvão M, Carlo A et al. Staplerless lap gastric bypass. Obes Surg. 2005 ; 15:1001-1.

Fonte de financiamento: não há Conflito de interesse: não há Recebido para publicação: 11/11/2007 Aceito para publicação: 15/02/2008 